Psychiatrists' views on Peer Support Workers

\title{
"Very much evolving": a qualitative study of the views of psychiatrists about Peer Support Workers
}

\section{$\underline{\text { Abstract }}$}

Background: Mental health services continue to develop service user involvement, including a growth in employment of Peer support workers (PSWs). Despite the importance of the views and attitudes expressed by psychiatrists, this topic has not previously been studied.

Aims: To gain insight into the views and attitudes psychiatrists have about peer support workers.

Methods: A qualitative study based on semi-structured interviews with 11 psychiatrists in the East of England.

Results: Psychiatrists were broadly positive and supportive of PSWs. Interviewees could anticipate a range of possible benefits of employing PSWs, but also had concerns regarding their implementation and management. There was a lack of clarity and consistency between interviewees about what the exact role of a PSW might involve.

Conclusion: This study provides insights into how PSWs are perceived by psychiatrists. While broadly positive attitudes exist, the research highlights certain challenges, particularly role ambiguity.

Declaration of interests: None to disclose

Keywords: Consumer participation, peer support workers, attitudes, psychiatrists 


\section{Psychiatrists' views on Peer Support Workers}

\section{Introduction}

The concept of peer support emerged from the survivor movement whereby individuals with lived experience of mental illness supported others in recovery (Slade et al 2014). Although only expanding recently in the UK, various forms of peer support workers (PSWs) are well-established internationally. In Australia individuals called 'consumer consultants' were initially employed nearly twenty years ago. In the USA certified peer specialists (CPS) were introduced from 2001 (Salzer et al 2010). PSWs are also found in New Zealand and across Europe.

UK Trusts employ service users in a range of roles including as trainers, mentors, recovery planning, recovery colleges and leading in social inclusion (Repper et al 2013). Most of these roles in the peer workforce are distinct from PSWs, because they do not involve direct one-to-one support. All PSWs will have personal experience of a mental health condition and are employed with the aim of sharing their recovery journey to motivate and encourage others. PSWs provide both practical help and hope to service users by promoting self-efficacy, modeling recovery and sharing coping strategies (Lloyd-Evans et el 2014).

Research findings into the evidence base of PSWs remain inconsistent. In a review of eighteen trials regarding PSWs, Lloyd-Evans et al (2014) found little evidence to demonstrate that the introduction of PSWs was associated with positive effects on hospitalization, overall symptoms or satisfaction with services, due to a lack of follow up. However, in a review of literature Repper and Carter (2011) conclude that PSWs can lead to a reduction in admissions and that they have the potential to encourage recovery-focused changes in services. 
Similarly, Gillard and Holley (2014) conclude that there is substantial qualitative evidence that demonstrate benefits at an individual level, with a smaller evidence base for impact on service use and costs.

The concept of PSWs is now commonly promoted in UK recovery literature with a considerable increase in the prevalence of PSWs since 2010 (Lloyd-Evans et al 2014). From 2011, the Centre for Mental Health and the NHS Confederation Mental Health Network have been promoting the "Implementing Recovery through Organisational Change" (IMROC) programme within the NHS, whom recommend PSWs (Repper et al 2013). Many UK health trusts are now employing PSWs, including Nottinghamshire Healthcare NHS Trust, Central and North West London NHS Foundation Trust, and also Cambridgeshire and Peterborough NHS foundation trust, which employed up to 80 such individuals from the outset (Basset et al 2010). The Trust which is the site of this study first employed PSWs in October 2014. Currently employing twenty-three PSWs, it plans to continue to expand upon this number. In this trust PSWs first undergo a 12-week training programme before beginning work.

\section{Professional attitudes}

Professional attitudes towards the peer workforce are likely to be important in the implementation and success of PSWs. Prior research has focused on healthcare professionals' attitudes towards broader 'service user involvement', demonstrating generally positive attitudes (Anthony \& Crawford 2000; McCann et al 2008). However, such research has also highlighted areas of concern regarding the effectiveness of service user involvement including unsuitable 


\section{Psychiatrists' views on Peer Support Workers}

patient characteristics and lack of ability or knowledge (Anthony \& Crawford 2000; Soffe et al 2004).

In a study exploring peer worker roles, Gillard et al (2014) interviewed 89 people from services involved in employing peer workers. They concluded that there was widespread evidence of support for peer workers throughout the organisations including at the highest organisational levels. Gillard et al 2014 recognised the importance of shared understanding of peer worker roles, but found mixed evidence that it existed - particularly in the NHS. Some coworkers in the NHS felt they should not be supporting peer workers or views them as an extra burden on their workload (Gillard et al 2014). Similarly, in an evaluation of a PSW pilot scheme it was found that some staff remain resistant to the PSW concept (McLean et al 2009). Various recommendations were made to aid the establishment of PSWs, including strong support from senior management and psychiatry; clarity over their role; full involvement in team; equal treatment as an employee; and nationally recognised formal training (McLean et al 2009).

The attitudes of psychiatrists towards PSWs is unclear. Due to psychiatrists' dominant role within mental health services, their views are likely to be particularly influential in the success of this innovation. Summers (2003) found that psychiatrists' attitudes to service user involvement in general cluster into three main groups: optimists (viewing service users as collaborators); rationalists (collaboration to a certain degree); and sceptics (service users have little to contribute). However, it is not known if this model of attitudes extends 


\section{Psychiatrists' views on Peer Support Workers}

to PSWs. This paper therefore aims to explore the views and attitudes of a sample of psychiatrists specifically towards PSWs.

\section{Methods}

Eleven psychiatrists working in the East of England were interviewed between January and March 2015. Interviewees were recruited opportunistically using existing contacts held by the researchers, and through approaching attendees at a mental health study day. This sampling approach was deemed appropriate, as the aim was to gain general insight into this group rather gather views which were representative of all psychiatrists. Nine of the interviewees had no previous contact with the lead researcher, one had limited previous contact and one had teaching contact. Interviews took place at either participant's workplaces or on university premises. Interviews lasted from 27 to 56 minutes and averaged 39 minutes. Interviews were semi-structured, exploring: experiences, definitions, advantages and disadvantages of different types of user involvement, in particular PSWs. This approach allowed pre-set questions to be asked whist exploring further upon answers given. Questions were developed from an extensive literature review on service user involvement.

All interviews were conducted by the same researcher to ensure similar style, structure and pace. A reflexive journal was utilised to promote awareness of the researcher's values and interests, including prior preconceptions and reflections on each interview. This was also used to note any non-verbal observations from interviews. 
A thematic analysis approach was employed, informed by Braun and Clarke (2006), to identify, analyse and report patterns within the data set. This approach was chosen as it is particularly suited to interpreting the conceptualisation of a phenomenon by a specific group (Joffe 2012). Using NVIVO as a data management tool, the lead researcher generated initial codes and sub-codes (Table 1) in a systematic fashion, which were then grouped into potential themes. Subsequently two other members of the research team reviewed these themes in relation to both the codes and the entire data set. Through this process the research team defined the following themes; The place of lived experience in mental health services, The contribution of the PSW, The impact of work on the PSW, Role ambiguity and PSW's and team dynamics.

\section{Sample}

Most were qualified medical practitioners at consultant level; one was a speciality registrar and another was a core trainee. They had an average of 15 years experience in psychiatry. Seven respondents worked in general adult services, but specialities of old age, forensic, early intervention, learning disabilities, young persons' mental health, and child and adolescent mental health services (CAMHS) were all represented. Seven interviewees were female and four male. All were familiar with the term 'PSW'. Two had worked with PSWs in their teams, four had other experiences of PSWs such a meeting them or attending an educational event on PSWs, three had no direct experience with PSWs and two did not disclose their experience of PSWs. 


\section{$\underline{\text { Results }}$}

\section{The place of lived experience in mental health services}

The vast majority of interviewees felt that PSWs could offer a new type of support to service users. Many referred to PSWs having a kind of authenticity; as being more approachable, and therefore able to offer something different from other staff:

"The PSW can make a big difference and they have a very unique role, they can do things that none of us with our qualifications and experience can do" (7)

Interviewees generally regarded lived experience of mental illness as a positive contribution to mental health services. Many participants referred to empowerment of service users, suggesting that initiatives led by service users tended to be more acceptable to them. Others spoke of the PSW role aiding in recovery, for example by helping with reducing stigma, living a satisfying life and looking at treatment and illness in a different way:

"giving someone ownership of their illness that....empowers them to hopefully take a more active role in the management of their illness and the management of their recovery....by being empowered to be engaged and taking ownership over their illness they suddenly for the first time....were able to move on..”(1)

However, many also questioned the ways in which services tried to employ lived experience. For example, one participant suggested that there was not necessarily a need for PSWs but instead for healthcare workers being open about their lived experience. However, many participants felt certain risks were 


\section{Psychiatrists' views on Peer Support Workers}

associated with them sharing their own experience of illness, for example stigma, maintaining professional relationships and a sense of isolation or standing out:

"I think the only thing that would worry me is that my patients need to feel that I am well and how that might be received by them because what I never want to convey to patients is that I need to be looked after in any way" (4)

Overall, there was optimism from respondents, who expected to work with growing numbers of PSWs, depending on the initial success:

"So when we get our PSW, if that goes really well I think we'll be begging for another one, you know it depends how that experience goes is the truth... if it's a disaster it will just dissolve as one of those ideas....I don't think it'll ever become really big but you know I think it'll you'll probably get a few more workers per team"' (8)

\section{The contribution of the peer support worker}

The majority of interviewees knew little about the training and selection of PSWs but assumed they would have fairly comprehensive training before beginning their post. Many felt careful selection was important. Some participants suggested PSWs might be helpful in the feedback and development of services:

"they have the actual experience of having been through the service so they know the challenges that that can bring and how people maybe get the better out of the services and may be able to think about again what is it you can do to help yourself, you know what it is that's going to help." (2)

It was also suggested that PSWs could be a cost effective measure;

"I guess it could be a cost effective way of aiding peoples recovery of giving people control of their own illness which is all really good. It might reduce the need for admissions and to be seen for secondary services if you've got better management and better support networks. 


\section{Psychiatrists' views on Peer Support Workers}

People when they've recovered that still got access to people but not having to come right into services" (8)

However some highlighted risks if PSWs were just treated as a cost saving measure, particularly the danger of exploitation:

“you can't just run on the fact that they have been unwell and can't work in the public sector so will do some volunteer work in the service and then actually the service is relying on you to do stuff, that's not a good position to be in." (6)

This respondent appeared unaware that PSWs are paid staff, albeit at lower rates than healthcare professionals.

There was also concern that services might not be fully able to support PSWs or get the most out of them:

"There's quite a big gap in the thinking I think possibly because teams are constantly at the moment, and have been over the past few years, in flux and under staffed anyway and so it doesn't give anyone the space to sort of think about that. Everyone's just sort of running day to day and you could kind of see that they could easily be dragged into just, like the rest of us, just managing the crisis of the day and is that the best use and are we going to just wear them out?”(2)

In an evidence-based service, another interviewee highlighted the challenge of how to measure the outcomes of PSWs:

"the outcomes for how you measure the recovery college or PSW are actually achieving what they set out to achieve are difficult to define" (6)

\section{The impact of work on the peer support worker}

Many interviewees referred to the personal benefits a PSW might find in the role. Two participants cited access to a career as an example. However one participant 


\section{Psychiatrists' views on Peer Support Workers}

went further, challenging the low expectations both service users have of themselves, and that health workers may have of service users:

\footnotetext{
"she (service user) said "I can't be a nurse I am mad" and I said well you are getting there you are on the way to recovery. She looked at me like I was bonkers in thinking about a career in something she was so evidently good at. It's interesting I was thinking about how patients have such low expectations of themselves and that is so difficult to reverse. And that could be a role for PSW" (4)
}

The majority of interviewees felt PSWs would need to carefully manage the use of their lived experience when working with service users. This involves potential conflicts in the change in relationship from doctor-patient to coworker. Generally it was felt that PSW should not work in the service where they had been treated. Many noted the personal challenge for PSWs in balancing their emotional response and maintaining the necessary boundaries when working with service users:

"I think it is challenging for them as they have to balance the role of not disclosing to much or getting caught up to much in their own story but using things that may be helpful” (11)

A concern shared by the majority was that the PSW could become ill. Most didn't view this as a barrier to employing PSWs. but instead something that needed careful consideration in the implementation of service user involvement. While there was particular emphasis on PSW illness due to the prerequisite of this for the role, some participants noted that illness is a concern for any employee. In particular, most respondents had concerns that PSWs might find the job stressful or triggering: 


\section{Psychiatrists' views on Peer Support Workers}

“Our support worker has gone off sick, very quickly. And so I think there's lots of issues which we can't pretend don't exist about that and how that is managed and how that is dealt with in the future" (2)

The majority stressed that PSWs must be well organised, with measures put in place to ensure their wellbeing. Most interviewees mentioned the need to care for and safeguard PSWs:

\footnotetext{
"but it's the organisation of that, and not just the organisation but making sure that's a safe process because you've got so many vulnerable people involved and you're responsible in a way if you started something like that" (8)
}

\section{Role ambiguity}

The majority of interviewees expressed confusion or lacked clarity over the role of PSWs. Most had limited experience of PSWs as they were in the early stages of being introduced to services. Some expressed a feeling that management had not thought through the role of PSWs. Many questioned what the difference was between support workers and PSWs, other than lived experience:

"Like I say it's very new and I think probably the challenge is working out what the role of PSWs is going to be within the teams, I think that's still very unclear as things stand....what is it that they should be doing that is different from a normal support worker or should they be the same as a support worker. I think it's not really been thought about” (2)

Two interviewees suggested a difference in the offering of emotional versus practical support by PSWs and support workers retrospectively:

"I expect they have a different remit, the support workers on our ward their role is very much in supporting patients with activities of daily living. But for PSW I think it's more about emotional support in recovery." (4) 
Although most lacked a clear understanding of the PSWs' role many suggested ideas of what a PSW might do, including: referring service users to recovery colleges; reducing stigma; instilling hope; acting as role models; teaching; offering one to one support; offering support in getting employment; and organising service user groups. Ideas of what a PSW does were sporadic amongst interviews: the only consistent thought between all interviews was that PSWs would use their lived experience of mental illness to support service users, some suggesting this would inspire hope:

"I guess what their role is to carry on doing some of the support work....but being explicit about their role as somebody who is an ex service user, so they may be able to use that dimension of their experience is a positive way with how they interact with people" (11)

\section{Peer Support Workers and team dynamics}

Three interviewees expressed a need for the multi-disciplinary team to understand and have clarity over the role of a PSW:

"if we were very clear, as with every clinician....to know what their roles are, for them to be clear about their role, so they don't feel overloaded or having to take on more than what would be expected of them" (10)

The majority acknowledged that having a previous service user as a member of their working team might pose difficulties, however it was felt that this is something teams could adjust to. Two participants saw no difficulty in a PSW integrating into a team. But two interviewees expressed a 'them and us' attitude or experience of PSWs, i.e. seeing them as not fully integrated into a team: 


\section{Psychiatrists' views on Peer Support Workers}

“it was very clear she (PSW) had also been a patient initially they couldn't really accept her as one of them so neither was she a patient and neither was she staff, she was kind of on the outside....for the team eventually they all got to know each other and it worked really well."

Three interviewees suggested that PSWs can alter the dynamics of a team, helping them view mental illness in a different way. One respondent raised the potential problem of a PSW coming from one particular perspective or agenda:

"One of the difficulties I have encountered with have service user involvement at every level is you can sometimes get service users who have a single sort of issue and they can only see things from their own perspective. So whilst trying to accommodate individuals like that it can sometimes prove disruptive to the process" (1)

\section{Discussion}

It is important to understand the attitudes of healthcare professionals as they can have significant impact on the success or failure of service user involvement initiatives (Gordon 2005). Amongst the group of psychiatrists interviewed, attitudes were broadly positive towards PSWs. However positive attitudes may mask a number of specific uncertainties regarding how they are implemented and what their exact role is within existing services.

A range of benefits of PSWs were noted, mainly focussing on the benefits for service users, including accessing a career, challenging low expectations and empowerment. Coinciding with previous research regarding broader service user involvement, wider benefits were also noted including reducing stigma, cost 


\section{Psychiatrists' views on Peer Support Workers}

effectiveness and the feedback to and development of services (Anthony \& Crawford, 2000; McCann et al, 2008; Robert et al, 2003; Rutter et al 2004).

Findings are also consistent with Summers (2003), who identified psychiatrists' support for service user involvement as likely concealing a range of attitudes. The range of viewpoints within this study could also be classified into Optimists, Rationalists and Sceptics as Summers (2003) noted. In contrast to previous findings, there was no evidence within this sample to suggest that professionals were resistant to view service users as experts, the vast majority valued lived experience (Tait \& Lester, 2005; Simpson \& House, 2003).

However, questions were raised about how PSWs would utilise their lived experience while also balancing emotional responses and boundaries. This is a common concern about PSWs (Repper and Carter 2011) and can be managed by adequate training and supervision (Davidson et al 2006). Gates and Akabas (2007) describe 'role conflict and confusion' on two levels; for the individual PSWs being both recipients and providers of services and for co-workers in terms of changing relationships with service users. Gillard et al (2014) warns of overformalisation of the peer worker role, which may inhibit the forming of peer-based relationships, suggesting a need to protect their unique position and not to overly identify as staff.

A significant theme throughout interviewees was role ambiguity. Many participants were unable to clearly define the PSW role, offering conceptual definitions, such as use of lived experience, as opposed to practical definitions of 


\section{Psychiatrists' views on Peer Support Workers}

their job role. This may be due to the limited first-hand experience of the sample and may reflect the Trust's failure to adequately prepare staff for the introduction of PSWs.

This role ambiguity may have a significant impact on the future success of PSWs. Gillard et al (2014) emphasised the importance of shared understanding of peer worker roles in facilitating successful role adoption. Role ambiguity may also inhibit the organisational flexibility required to accommodate PSWs and their distinct qualities (Gillard et al 2014). It was generally felt that PSWs would change the dynamics of existing teams, which was met with a mixed response.

Concerns generally focussed around the felt need to safeguard and care for PSWs. These well intentioned concerns may conceal stigmatising attitudes with some displaying a 'them and us' attitude towards PSWs; both in terms of their role within the team and in terms of vulnerability. Many interviewees felt PSWs may be too vulnerable, easily overloaded and prone to relapse. It can be argued that PSWs can harness any vulnerability to build relationships with other service users. These concerns may also be due to limited knowledge of the recruitment and training of PSWs, which may be more apparent in a service that is relatively new to PSWs.

The findings of this study are particularly relevant in the context of the recommendations made by McLean et al (2009). The success of PSWs requires strong support from senior management and psychiatry, which may be hindered by the lack of clarity concerning the PSW role and their place within existing 


\section{Psychiatrists' views on Peer Support Workers}

services, as expressed by this sample. Such issues can be addressed through educating staff, employing strategies to combat stigma and providing on-going support to staff to maximise inclusion (Gates and Akabas 2007). McLean et al (2009) also emphasised the importance of treating PSWs as any other employee and part of the team. This approach was only partly evident amongst this sample and seems to be something that will be crucial for the future success of PSWs.

Successful examples of peer workers have been evident in organisations that have been able to be flexible and where PSWs had been established for longer (Gillard et al 2014). Gillard and Holley (2014) emphasise the need for careful consideration of the organisational issues around peer worker roles. For PSWs to be successful shared expectations, understanding of the distinctive role of PSWs and a supportive organisation is required. While there is controversy surrounding the evidence base of PSWs it is clear that their success depends upon strong organisational support of which psychiatrists hold an influential positon.

\section{Limitations of study}

The relatively small sample size of the study in a limitation. Participants were also recruited via referral sampling and this may not have resulted in a wide cross-section of stances, although the results do demonstrate a range of attitudes including both optimists and sceptics. Although the vast majority of interviewees were not known to the researcher the nature of the interview topic may have resulted in participants concealing specific opinions. It is also worth noting that expressed opinions may not be reflected in individuals' actions. 


\section{$\underline{\text { References }}$}

Anthony, P. \& Crawford, P. (2000). Service user involvement in care planning: the mental health nurse's perspective. Journal of Psychiatric and Mental Health Nursing, 7, 425-434.

Basset ,T., Faulkner, A., Repper, J. \& Stamou, E. (2010). Lived Experience Leading the Way: Peer Support in mental health. Together UK.

Braun, V. \& Clarke, V. (2006). Using thematic analysis in psychology. Qualitative research in psychology, 3:2, 77-101.

Davidson, L., Chinman, M., Sells, D. \& Rowe, M. (2006). Peer support among adults with serious mental illness: A report from the field. Schizophrenia Bulletin, 32, 443-450

Gates, L.B. \& Akabas, S.H. (2007). Developing strategies to integrate peer providers into the staff of mental health agencies. Journal of Administration and Policy in Mental Health and Mental Health Services Research, 34, 293-306.

Gillard, S., Edwards, C., Gibson, S., Holley,J. \& Owen, K. (2014). New ways of working in mental health services: a qualitative, comparative case study assessing and informing the mergence of new peer worker roles in mental health services in England. Health Services and Delivery Research, 2 


\section{Psychiatrists' views on Peer Support Workers}

Gillard, S. \& Holley, J. (2014). Peer workers in mental health services: literature overview. Advances in psychiatric treatment, 20, 286-292.

Gordon, S. (2005). The role of the consumer in the leadership and management of mental health services. Australasian Psychiatry, 13, 362-365.

Joffe, H. (2012). Thematic Analysis. In Harper, D. \& Thompson, A. (Eds)

Qualitative research methods in mental health and psychotherapy: A guide for student and practitioners. Chichester: Wiley-Blackwell, pp209-223.

Lloyd-Evans, B., Mayo-Wilson, E., Harrison, B., Istead, .H, Brown, E., Pilling, S., Johnson, S. \& Kendall, T. (2014). A systematic review and meta-analysis of randomised control trials of peer support for people with severe mental illness. BMC Psychiatry, 14, 39

McCann, T.V., Baird, J., Clark, E. \& Lu, S. (2008). Mental health professionals' attitudes towards consumer participation in inpatient units. Journal of Psychiatric Mental Health Nursing, 15, 10-16.

McLean, J., Biggs, H., Whitehead, I., Pratt, R. \& Maxwell, M. (2009). Evaluation of the delivering for mental health peer support worker pilot scheme. Scottish Government social research.

Repper, J. \& Carter, T. (2011). A review of the literature on peer support in mental health services. Journal of Mental Health, 20, 392-411 
Repper, J., Aldridge, B., Gilfoyle, S., Gillard, S., Perkins, R. \& Rennison, J. (2013). Peer support workers: Theory and Practice. ImROC briefing paper, Centre for mental health and mental health network, NHS confederation.

Robert, G., Hardacre, J., Locock, L., Bate, P. \& Glasby, J. (2003). Redesigning mental health services: lessons on user involvement from the Mental Health Collaborative. Health Expectations, 6, 60-71.

Rutter, D., Manley, C., Weaver, T., Crawford, M.J. \& Fulop, N. (2004) Patients or partners? Case studies of user involvement in the planning and delivery of adult mental health services in London. Social Science and Medicine, 58, 1973-84.

Salzer, M., Schwenk, E. \& Brusilovskiy, E., (2010). Certified peer specialist roles and activities: results from a national survey. Psychiatric services, 61:5, 520-523.

Simpson, E.L. \& House, A.O. (2003). User and carer involvement in mental health services: from rhetoric to science. British Journal of Psychiatry, 183, 89-91.

Slade, M., Amering, M., Farkas, M., Hamilton, B., O’Hagan, M., Panther, G., Perkins, R., Shephard, G., Tse, S. \& Whitley, R. (2014). Uses and abuses of recovery: implementing recovery-oriented practices in mental health systems. World Psychiatry, 13, 12-20. 


\section{Psychiatrists' views on Peer Support Workers}

Soffe, J., Read, J. \& Frude, N. (2004) A survey of clinical psychologists' views regarding service user involvement in mental health services. Journal of Mental Health, 13, 583-592.

Summers, A. (2003). Involving users in the development of mental health services: A study of psychiatrists' views. Journal of Mental Health,12, 161-174.

Tait, L. \& Lester, H. (2005). Encouraging user involvement in mental health services. Advances in Psychiatric Treatment, 11, 168-75. 